\title{
Correlative Analysis using FIB-ToF-SIMS and Atom Probe Tomography on Geological Materials
}

\author{
William D.A. Rickard ${ }^{1,2}$, Steven M. Reddy ${ }^{1,3}$, David W. Saxey ${ }^{1,2}$, Denis Fourgerouse ${ }^{1,3}$ and Arie van \\ Riessen $^{1,2}$ \\ 1. Geoscience Atom Probe, Advanced Resource Characterisation Facility \& John de Laeter Centre, \\ Curtin University, Perth, Australia. \\ 2. Department of Physics and Astronomy, Curtin University, Perth, Australia. \\ 3. Department of Applied Geology, Curtin University, Perth, Australia.
}

Atom probe tomography (APT) has recently been applied to geological materials [1,2]. Complex microstructures such as low angle boundaries and phase boundaries are often targeted for analysis as they can yield important information about geological processes.

In order to define a robust framework and support findings from APT it is increasingly important to correlate the results with other analyses. Surface analyses such as imaging (SE, BSE), elemental mapping (EDS) and microstructural mapping (EBSD) provide complementary information at longer length scales, though in many cases can be difficult to directly correlate with APT results due to the much smaller analysis region. High-resolution studies of sample tips by imaging (TEM), elemental mapping (TEM-EDS), and microstructural mapping (TKD), have been demonstrated to provide useful information specific to the APT analysis region.

Here we present results on a novel complementary analysis technique which has a number of benefits for APT data analysis. A Tescan Lyra FIB-SEM installed at Curtin University has been fitted with an orthogonal Time of Flight Secondary Ion Mass Spectrometer (ToF-SIMS). Details of the ToF-SIMS can be found in [3]. This combination enables the FIB-SEM to be used for high precision site-selective atom probe sample preparation and for in-situ TOF-SIMS analyses.

FIB-ToF-SIMS is used for surface and depth resolved elemental mapping as well as providing isotopic information. Significant benefits in light element and trace element mapping can be achieved when compared to x-ray based techniques. The small information volume (approx. the same size as the FIB probe, e.g $<25 \mathrm{~nm}$ ) allows for high spatial resolution elemental maps (Fig. 1) which is particularly useful for light and low concentration elements. When low beam currents are used, only a few tens of nanometers of material is removed from the sample surface allowing the mapped region to be prepared for subsequent atom probe analysis.

Another significant benefit is that the ToF-SIMS produces mass/charge spectra that can be directly correlated with mass/charge spectra from atom probe analyses. Different ionisation conditions between the two techniques mean that in some cases ambiguous peaks can be identified by comparing the two data sets. Comparison of a ToF-SIMS analysis with an atom probe analysis from an olivine sample (Fig. 2) shows that the ToF-SIMS spectra contains less ions with a double charge state. The ToF-SIMS can be configured to collect either positive or negative ions, thus providing additional versatility when targeting specific elements. 
References:

[1] J Valley et al, Nature Geoscience 7 (2014), p. 219-223.

[2] S Piazolo et al, Nature Communication 7 (2016), p. 1-7.

[3] D Alberts et al, Instrumentation Science \& Technology 42 (2014), p. 432-445.
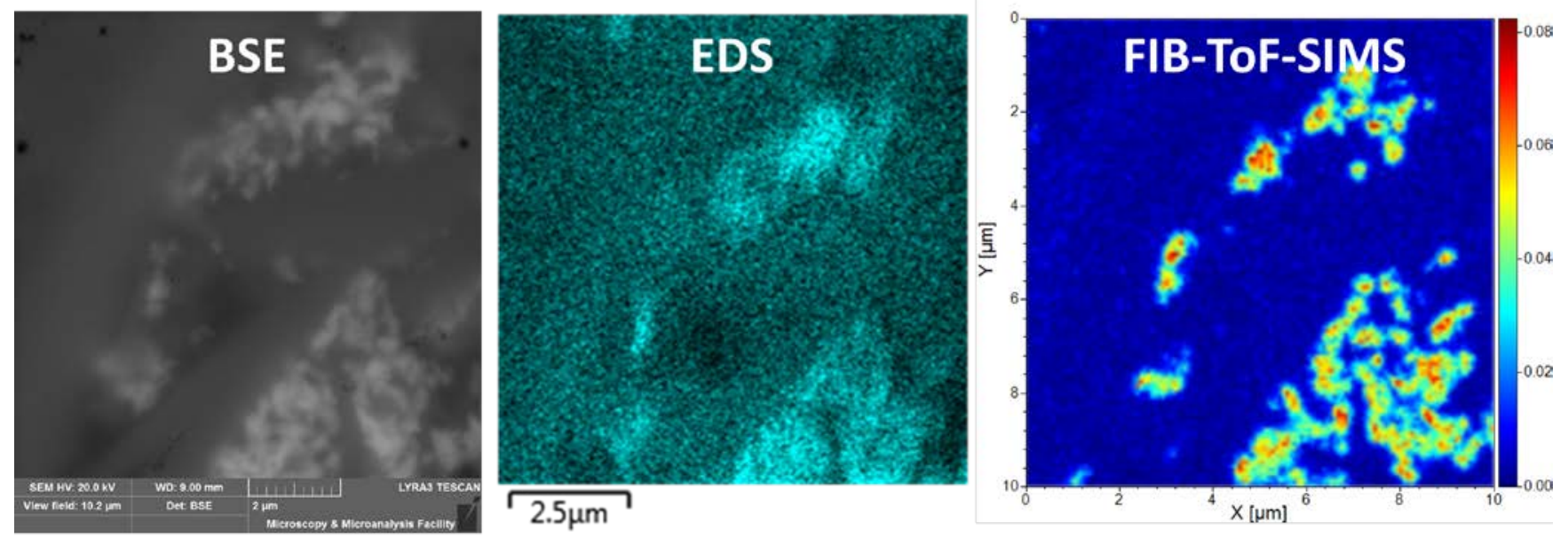

Figure 1. Backscattered (BSE) image with corresponding energy dispersive x-ray spectroscopy (EDS) and FIB-ToF-SIMS elemental maps for oxygen from a coal sample. The high spatial resolution of the ToF-SIMS maps allows for more accurate APT targeting than can be achieved with EDS analyses.

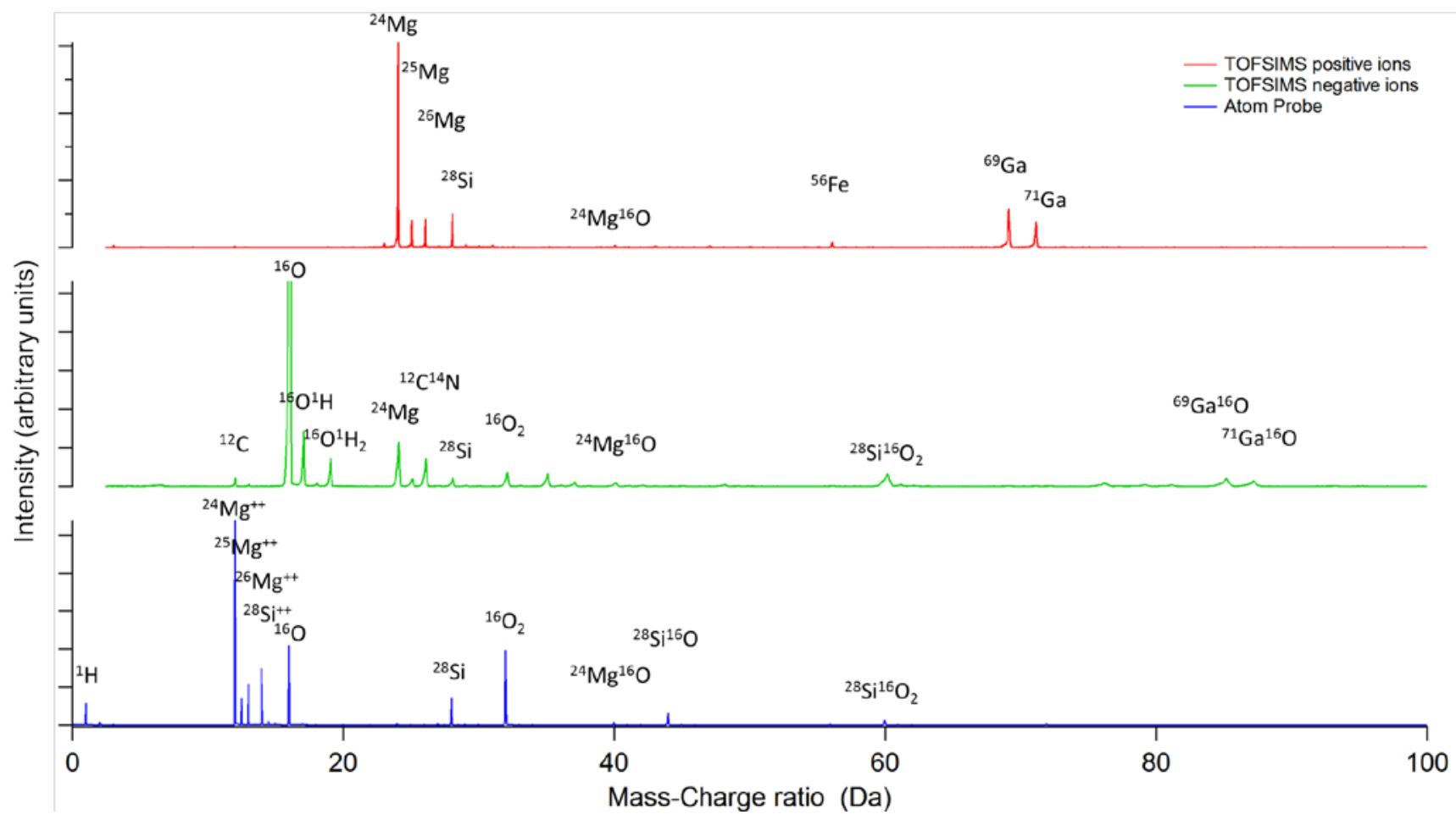

Figure 2. Comparison between Atom Probe and ToF-SIMS mass spectra from a San Carlos Olivine. Positive and negative ToF-SIMS spectra are displayed. Gallium peaks are from the FIB primary ion source. 